\title{
Caracterização da hidrólise parcial enzimática de amido isolado de abacate utilizando técnicas termoanalíticas e microscopia
}

\author{
L. C. Malucelli ${ }^{1}$, C. F. Lemos ${ }^{1}$, M. A. S. C. Filho ${ }^{1}$, I. M. Demiate, \\ E. Schnitzler ${ }^{2}$, L. G. Lacerda ${ }^{1,2^{*}}$
}

${ }^{1}$ Universidade Positivo - UP - Rua Prof. Pedro Viriato Parigot de Souza, 5300, CEP 81280-330, Curitiba-PR, Brasil.

${ }^{2}$ Universidade Estadual de Ponta Grossa-UEPG - Av. Carlos Cavalcanti, 4748, CEP 84030-900, Ponta Grossa-PR, Brasil

Received 03/06/2014; accepted 21/07/2014

Available online 30/12/2014

\begin{abstract}
Resumo
O amido é um biopolímero presente em raízes, frutos, tubérculos e sementes, se configurando como fonte de armazenamento de energia. Como matéria-prima, pode ser utilizado em diversos setores, como: indústria química, de papel, farmacêuticas e alimentos, entre outros. O uso de amidos alternativos é preconizado para o que acima é descrito e o amido de abacate, por se tratar de um subproduto, se insere como inovação. No presente trabalho, a hidrólise parcial do amido de abacate foi avaliada em diferentes tempos de tratamento, tendo-se utilizado termogravimetria, análise térmica diferencial (TG-DTA) e calorimetria exploratória diferencial (DSC), bem como microscopia óptica. Os resultados obtidos mostram que, com o ataque enzimático, há alteração estrutural e morfológica dos grânulos.
\end{abstract}

Keywords: Amido, hidrólise parcial, amilase fúngica

\section{Introdução}

O Brasil é um dos maiores produtores mundiais de abacate (Persea americana). Trata-se de uma produção que gerou em torno de 166 mil toneladas para o ano de 2011 [1]. Embora a maior parte da produção seja exportada, o consumo interno desta fruta tem aumentado de forma considerável, pois se trata de um alimento de elevado valor nutricional apreciado na culinária e com ampla aplicação na indústria farmacêutica. $\mathrm{O}$ abacateiro é originário do México e América Central, pertence à família Lauraceae e gênero Perseal [2]. Apresenta excelente produtividade por unidade de área de cultivo e trata-se de uma fruta constituída basicamente de polpa e caroço.

A polpa contém vitaminas lipossolúveis, escassas na maioria das frutas tropicais, além de outras vitaminas, como A e B. Possui entre 5 e $35 \%$ de óleo e teor de açúcar de 3 a $8 \%$ [3]. O óleo extraído da polpa possui ampla utilização nas indústrias cosmética e farmacêutica, além de ser utilizado como substitutivo do óleo de oliveira, uma vez que apresenta grande semelhança constituinte, principalmente no que diz respeito a composição de ácidos graxos $[\underline{4}, \underline{5}]$. O caroço é constituído, basicamente, de fibras e elevado percentual de amido. Chega a dividir com a polpa a metade do peso da fruta, sendo atualmente descartado, ou seja, sem utilidade específica.

O amido, presente na semente, é um componente orgânico abundante na natureza. Trata-se de um carboidrato sintetizado pelos vegetais e utilizado como reserva energética. Sua estrutura é constituída por dois polissacarídeos, amilase e amilopectina, polímeros de glicose, que, de acordo com a procedência do amido, variam percentualmente []․ O uso do amido nativo é muito diversificado e no Brasil ocorre, principalmente, em indústrias alimentícias, além de outras, como, por exemplo, metalúrgica, mineração, construção, cosmética, farmacêutica, papel e papelão, têxtil, etc.. A demanda crescente da indústria infere enorme pressão sobre as fontes oficiais de amido, daí a necessidade da busca contínua por outras fontes economicamente viáveis []].

De maneira geral, de acordo com a literatura, as propriedades do amido natural são limitadas para uso direto em indústrias. Comumente, a modificação dos grânulos é feita para melhorar a textura das pastas ou géis, aumentar adesividade e melhorar a formação de filmes, entre outros. Assim, alterações por métodos físicos, químicos e biológicos são frequentemente realizados nos amidos para alterar suas propriedades naturais e ampliar sua utilização industrial [7]. As razões que levam à modificação incluem: modificar as características de cozimento (gomificação), diminuir a retrogradação e a tendência das pastas formarem géis, aumentar a estabilidade das pastas frente ao congelamento e descongelamento, melhorar a transparências das pastas e géis, textura e a adesividade, além da formação de filmes e adicionar grupamentos hidrofóbicos e introduzir poder emulsificante [8]. Alterações nas propriedades tecnológicas dos amidos podem ser obtidas por processos físicos, tais como tratamento térmico, exposição a radiações, ou por processos químicos nos quais se empreguem reagentes específicos para alterar as estruturas das macromoléculas componentes do amido. Ainda há a possibilidade de serem empregados processos enzimáticos [9].

Diante deste quadro, o mercado de amido vem se aperfeiçoando, levando à busca de produtos com características específicas que atendam às exigências da

\footnotetext{
* Corresponding author: Tel.: +55 (00) 0000-0000

E-mail address: luizgustavo@up.com.br (L. G. Lacerda)
} 
indústria. A produção de amidos modificados é uma alternativa que vem sendo desenvolvida há algum tempo, com o objetivo de superar uma ou mais limitações do amido nativo e assim aumentar a utilidade deste polímero nas aplicações industriais $[\underline{10}, \underline{11]}$.

\section{Experimental}

\subsection{Materiais e métodos}

As sementes dos frutos de $P$. americana foram obtidas através da remoção do revestimento exterior e do mesocarpo do fruto. Os núcleos foram cortados em pequenos pedaços e embebidos em uma solução $0,075 \%$ de $\mathrm{Na}_{2} \mathrm{~S}_{2} \mathrm{O}_{5}$, durante $24 \mathrm{~h}$, para evitar a degradação oxidativa. As peças pré-hidratadas foram trituradas com a finalidade de se obter um material degradado fisicamente e homogêneo, utilizando-se um liquidificador em potência alta, durante 5 minutos. Foi adicionada água destilada e a suspensão obtida foi deixada em repouso durante $12 \mathrm{~h}$, em geladeira, para a sedimentação completa dos grânulos de amido. Este procedimento foi repetido por 3 vezes. A fração amilácea permaneceu como precipitado e foi lavada com água destilada a temperatura de $15{ }^{\circ} \mathrm{C}$. A massa de amido obtida foi seca em estufa a vácuo durante $24 \mathrm{~h}$, a $50^{\circ} \mathrm{C}$.

Para o processo de hidrólise foram necessários erlenmeyers com a identificação no frasco do material a ser analisado e referência ao tempo de hidrólise em que foram submetidos. Foram adicionadas 0,0150 g de um complexo enzimático Distillase SSF (Genecor - EUA), segundo a recomendação da ficha técnica da mesma, considerando a massa de amido. As amostras foram identificadas, seladas e incubadas em agitação em temperatura de $30^{\circ} \mathrm{C}$ e $100 \mathrm{rpm}$. As amostras variaram quanto ao tempo de tratamento, de 12 h, 24 h e 36 horas de atividade da enzima, além da análise do amido de abacate sem tratamento enzimático. A cada $12 \mathrm{~h}$ foi feita a retirada de uma amostra para análise. A filtração, a vácuo, foi realizada para obtenção da fração sólida das mostras secas, que foram secas em forno de convecção por aproximadamente $12 \mathrm{~h}$.

As curvas termogravimétricas foram obtidas utilizando o equipamento TG 60 (SHIMADZU - Japan), através de pesagem prévia de amostras de aproximadamente $5 \mathrm{mg}$, em balança de precisão analítica. As amostras foram pesadas e acondicionadas em cadinhos de alumina previamente tarados na termobalança. As condições de análise foram as seguintes: razão de aquecimento de $10{ }^{\circ} \mathrm{C} \mathrm{min}^{-1}$; atmosfera de ar sintético com vazão de $100 \mathrm{~mL} \mathrm{~min}{ }^{-1}$; temperatura inicial de $30{ }^{\circ} \mathrm{C}$; temperatura final de $600{ }^{\circ} \mathrm{C}$.

As curvas DSC foram obtidas em equipamento DSC 60 (SHIMADZU - Japan). Foi feita a pesagem de amostras e seu devido acondicionamento em cadinhos seláveis de alumínio (SHIMADZU), em balança analítica em torno de $2 \mathrm{mg}$, seguido de dispersão em $6 \mu \mathrm{L}$ de água para cada amostra, com auxílio de micropipeta, de modo a permitir o evento de gelatinização. As condições de análise foram as seguintes: razão de aquecimento de $5{ }^{\circ} \mathrm{C} \min ^{-1}$; ar sintético como atmosfera; vazão de $100 \mathrm{~mL} \mathrm{~min}^{-1}$; temperatura inicial de $30{ }^{\circ} \mathrm{C}$; temperatura final de $100{ }^{\circ} \mathrm{C}$. As curvas TG-DTA e
DSC obtidas foram observadas no programa TA 60 (SHIMADZU - Japan).

As amostras de amido de abacate foram observadas em uma lupa estereoscópica (Olympus SZX9), composta por filtro polarizador e fotografada com o captador de imagens (MEDIA CYBERNETICS). As fotografias foram identificadas usando o programa IMAGE PRO PLUS. As amostras foram observadas em objetiva com aumento de 100X e 1000X, com auxílio de óleo de imersão.

\section{Resultados e Discussão}

A calorimetria exploratória diferencial (DSC) foi realizada para determinar as propriedades de gelatinização do amido. Suas curvas de gelatinização das amostras com e sem tratamento enzimático estão representadas na Figura 1. Conforme resultados fornecidos por DSC (Tabela 1) ocorreu uma variação de temperatura para início de processo de gelatinização entre as amostras tratadas de abacate, de modo que a amostra sem tratamento enzimático precisou de uma maior temperatura dentre as amostras para a transição de fase (pico).

Tabela 1: Valores obtidos através da calorimetria exploratória diferencial (DSC) com temperatura de início do processo, pico e valores de entalpia para amostras de amido de abacate (a) in natura, após (b) 12, (c) 24 e (d) 36 horas de hidrólise.

\begin{tabular}{ccccc}
\hline \multirow{2}{*}{ Amostra } & \multicolumn{4}{c}{ DSC de gelatinização } \\
\cline { 2 - 5 } & $\mathrm{T}_{\mathrm{o}} /{ }^{\circ} \mathrm{C}$ & $\mathrm{T}_{\mathrm{p}} /{ }^{\circ} \mathrm{C}$ & $\mathrm{T}_{\mathrm{c}} /{ }^{\circ} \mathrm{C}$ & $\Delta \mathrm{H}_{\mathrm{gel}} / \mathrm{J}^{\circ} \mathrm{g}^{-1}$ \\
\hline (a) & 67,15 & 70,35 & 75,14 & 8,89 \\
(b) & 66,69 & 70,76 & 73,09 & 9,01 \\
(c) & 63,67 & 67,25 & 72,18 & 12,93 \\
(d) & 64,59 & 68,13 & 73,66 & 12,75
\end{tabular}

(*) $\mathrm{T}_{\mathrm{o}}$ "onset" temperatura inicial, $\mathrm{T}_{\mathrm{p}}$ temperatura de pico, $\mathrm{T}_{\mathrm{c}}$ "endset" temperatura de conclusão, $\Delta \mathrm{H}_{\mathrm{gel}}$ entalpia de gelatinização.

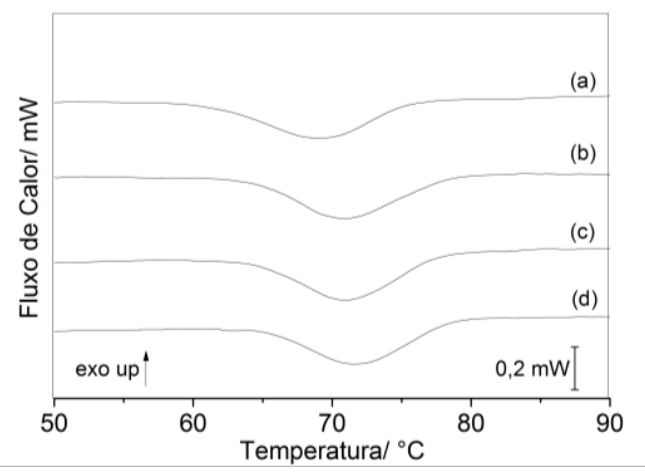

Figura 1: Curvas DSC ilustrando a gelatinização de amostras de amido de abacate (a) in natura, após (b) 12, (c) 24 e (d) 36 horas de hidrólise.

No estudo, observou-se que quanto maior é o tempo de tratamento das amostras, menor é a temperatura necessária para a ocorrência do evento de gelatinização, que pode indiciar uma alteração estrutural do grânulo. Outro ponto observado foi a ocorrência de um provável aumento da degradação do amido, tendo em vista que as amostras tratadas apresentaram valores mais elevados de energia 
envolvida na gelatinização do que a amostra não tratada. A entalpia entre amostras, para o evento de transição de fase, ocorreu com demanda crescente. Como comparação, estudos mostraram que a degradação por enzimas fúngicas resultou num aumento de entalpia frente ao aumento do tempo de tratamento das amostras, o que sugere um aumento do teor de cristalinidade, comparado ao inicial para as amostras em questão [11-13]. As curvas de TG-DTA de amido de abacate estão representadas na Figura 2 e as etapas - variação de massa, faixa de temperatura, picos e natureza térmica - são apresentadas na Tabela 2 .
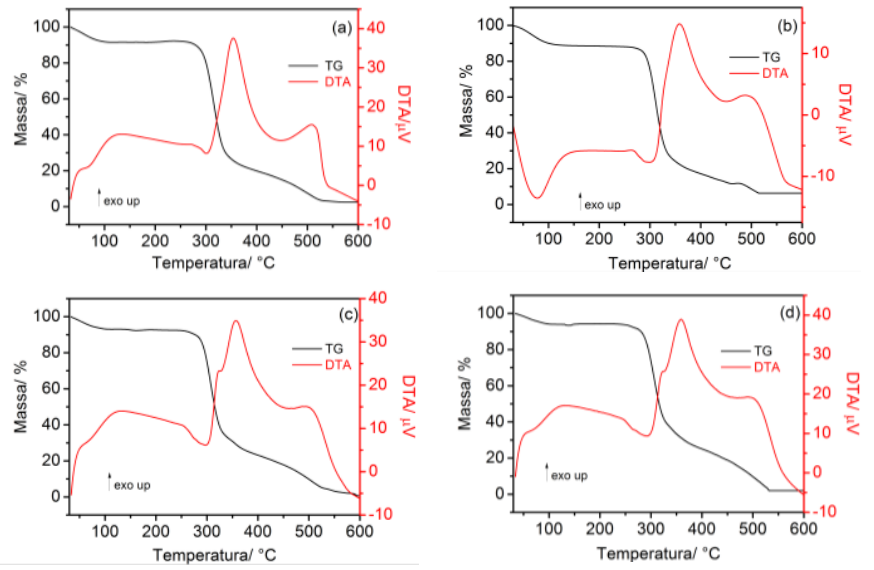

Figura 2: Gráfico TG-DTA do amido de abacate (a) in natura, após (b) 12, (c) 24 e (d) 36 horas de hidrólise.

Tabela 2: Resultados de TG e DTA: a) amido não tratado de abacate; b) hidrolisado de amido ceroso de milho de $12 \mathrm{~h}$; c) hidrolisado de amido ceroso de milho de $24 \mathrm{~h}$; d) hidrolisado de amido ceroso de milho de $36 \mathrm{~h}$.

\begin{tabular}{|c|c|c|c|c|}
\hline \multirow[t]{2}{*}{ Amostra } & \multicolumn{2}{|c|}{ TG Resultados } & \multicolumn{2}{|c|}{ DTA Resultados } \\
\hline & Etapa & $\Delta \mathrm{m} / \%$ & $\Delta \mathrm{T} /{ }^{\circ} \mathrm{C}$ & $\mathrm{Tp}\left({ }^{\circ} \mathrm{C}\right)$ \\
\hline \multirow[t]{5}{*}{ a) } & $1^{\circ}$ & 8,28 & $30,00-150,0$ & 73,1 (Endo) \\
\hline & Estabilidade & - & $150,0-298,6$ & - \\
\hline & $2^{\circ}$ & 65,90 & $283,2-379,5$ & 352,9 (Exo) \\
\hline & $3^{\circ}$ & 18,00 & $379,5-527,4$ & $507,0($ Exo $)$ \\
\hline & Perda total & 97,34 & $30,00-600,0$ & - \\
\hline \multirow[t]{5}{*}{ b) } & $1^{\circ}$ & 11,15 & $30,00-150,0$ & 78,1 (Endo) \\
\hline & Estabilidade & - & $150,0-278,0$ & - \\
\hline & $2^{\circ}$ & 74,69 & $278,0-457,7$ & 357,8 (Exo) \\
\hline & $3^{\circ}$ & 4,47 & $457,7-508,5$ & 490,0 (Exo) \\
\hline & Perda total & 99,45 & $30,00-600,0$ & \\
\hline \multirow[t]{5}{*}{ c) } & $1^{\circ}$ & 7,33 & $30,00-150,0$ & 75,5 (Endo) \\
\hline & Estabilidade & - & $150,0-282,8$ & - \\
\hline & $2^{\circ}$ & 70,48 & $282,8-450,2$ & 359,3(Exo) \\
\hline & $3^{\circ}$ & 12,87 & $450,2-533,3$ & 502,9 (Exo) \\
\hline & Perda total & 99,60 & $30,00-600,0$ & - \\
\hline \multirow[t]{5}{*}{ d) } & $1^{\circ}$ & 6,94 & $30,00-150,0$ & 79,2 (Endo) \\
\hline & Estabilidade & - & $150,0-279,7$ & - \\
\hline & $2^{\circ}$ & 72,64 & $279,7-460,4$ & 359,0 (Exo) \\
\hline & $3^{\circ}$ & 15,80 & $460,4-501,7$ & 501,7 (Exo) \\
\hline & Perda total & 98,47 & $30,00-600,0$ & \\
\hline
\end{tabular}

$\Delta \mathrm{m}$ perda de massa $(\%), \Delta \mathrm{T}$ variação de temperatura, Tp pico de temperatura
As curvas TG foram usadas para definir e calcular a perda de massa de cada amostra em relação ao tempo, temperatura e atmosfera. Três intervalos característicos são observados nas curvas TG: inicialmente, em intervalo de 30 e $150{ }^{\circ} \mathrm{C}$, representado pela evaporação de água e compostos voláteis (pico endotérmico), seguido de estabilidade. Os intervalos restantes referem-se à degradação de matéria orgânica (amilose e amilopectina) e formação de cinzas. Os resultados obtidos foram semelhantes aos encontrados em estudos prévios, utilizando diferentes tratamentos e amidos de variadas fontes botânicas $[\underline{9}, \underline{13}, \underline{14}]$.

De acordo com a literatura, o tratamento térmico de amidos leva normalmente à sua despolimerização, quando a temperatura utilizada ultrapassa os $300{ }^{\circ} \mathrm{C}$, em atmosfera oxidativa. $\mathrm{O}$ amido sofre uma série de alterações irreversíveis, de modo que a alteração estrutural no polímero leva inicialmente à formação de pirodextrinas e, a temperaturas mais elevadas, a degradação de macromoléculas resulta na formação de levoglucosano, furfural e de produtos voláteis de menor massa molecular e, por fim, restam apenas cinzas $[\underline{11}, \underline{13}]$.

Também se verificou, no estudo, que os amidos tratados apresentaram teores mais elevados de cinzas restantes do que o amido sem tratamento, diferentemente do que observado em pesquisas recentes utilizando degradação parcial via ácida e utilizando bagaço de mandioca [13, 14]. As amostras sem tratamento apresentaram formato essencialmente alongado e superfície plana (Figura 3). As amostras com tratamento de 12,24 e $36 \mathrm{~h}$, respectivamente (Figura 4), apresentaram degradação gradativa, resultando em reduzidas porções dos grânulos em comparação ao estado inicial.
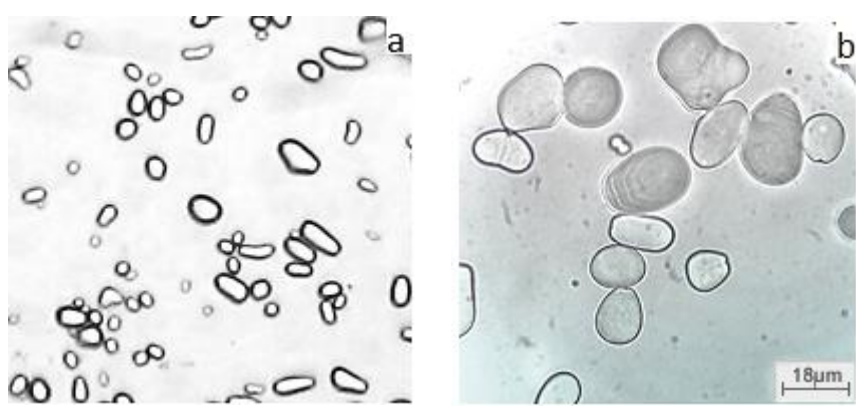

Figura 3: Microscopia dos grânulos de amido de abacate in natura com ampliação de 100X (a) e 1000X (b).
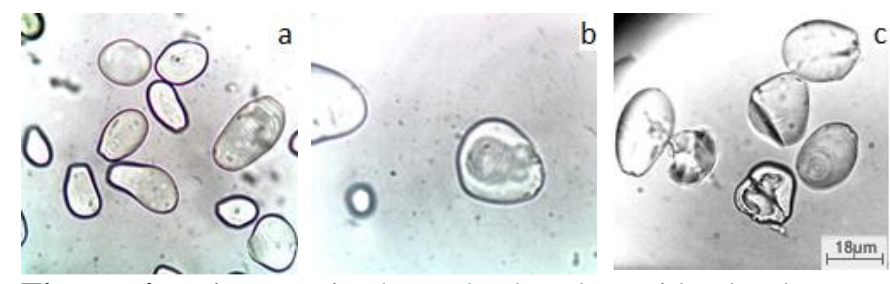

Figura 4: Microscopia dos grânulos de amido de abacate com tratamento enzimático após 12 horas (a), 24 horas (b) e 36 horas (c) com ampliação de 1000X.

Outros estudos apresentaram resultados morfológicos semelhantes $[\underline{9}, \underline{15}]$. De acordo com a literatura, a taxa de hidrólise de amostras de amido de abacate diminui com o aumento de sua taxa de cristalinidade. A erosão enzimática de grânulos de amido ocorreu principalmente em sua 
superfície $[\underline{16}, \underline{17]}$. O resultado da atividade enzimática dos grânulos foi comprovado por outros estudos anteriores, utilizando outros tipos de amido como o ceroso, além do de milho [18, 19]. Pesquisadores verificaram que a taxa de digestibilidade do amido é afetada por muitos fatores, dentre eles a fonte botânica do amido utilizado na degradação enzimática $[\underline{18}, \underline{20}, \underline{21}]$.

A literatura mostra que o tratamento enzimático de amido pode ser realizado de modo a obter maiores quantidades de amido lentamente digerível, o que é fonte de muitos estudos atuais no ramo da nutrição e suplementação alimentar [22].

\section{Conclusões}

O estudo proposto auxiliou numa melhor caracterização estrutural e de comportamento dos grânulos de amido de abacate sem tratamento e frente à hidrólise enzimática controlada. Para as curvas de DSC, observou-se crescente variação de energia requerida para transição de fase das amostras. Já em relação à temperatura de início do processo, as amostras de amido de abacate, com tratamento, apresentaram menor temperatura, o que pode ser um indicativo de uma maior alteração estrutural. As curvas TG e DTA apresentaram perda de massa em três estágios, representados por desidratação, decomposição e oxidação da matéria orgânica, respectivamente. Por fim, as micrografias oriundas de microscopia óptica auxiliaram no estudo do efeito do tratamento enzimático das amostras, de modo que foram obtidas porções reduzidas dos grânulos proporcionais aos intervalos de tempo de tratamento.

\section{Referências bibliográficas}

[1] Instituto Brasileiro de Geografia e Estatística. Anuário Estatístico do Brasil. 2012. [Visualizar Item] Acessado em 08 Jul 2014.

[2] Tango JS, Carvalho CRL, Soares NB. Caracterização física e química de frutos de abacate visando a seu potencial para extração de óleo. Rev. Bras. Frutic. 2004:26(1):17-23. [Google Scholar] [Visualizar Item] [CrossRef]

[3] Koller OC. Abacaticultura. Porto Alegre: UFRGS; 1992. [Google Scholar]

[4] Bleinroth EW, Castro JV. Abacate: Cultura, matéria-prima, processamento e aspectos econômicos. Campinas: ITAL; 1978. [Google Scholar]

[5] Turatti JM, Santos LC, Arima HK. Caracterização do óleo de abacate obtido por diferentes processos de extração. Bol. Ital. 1985:22:267-284. [Google Scholar]

[6] Builders PF, Numrum A, Mbah CC, Attama AA, Manek R. The physicochemical and binder properties of starch from Persea Americana Miller (Lauraceae). Starch/Staerke. 2010:62(6):309-320. [Google Scholar] [Visualizar Item] [CrossRef]
[7] Batista WP, Silva CEM, Liberato MC. Propriedades químicas e de pasta dos amidos de trigo e milho fosforilados. Cien. Tecnol. Alim. 2010:30(1):88-92.

[Google Scholar] [Visualizar Item] [CrossRef]

[8] Silva PL, Gomes AMM, Ricardo NPMS, Silva CEM. Caracterização fisico-química e reológica dos amidos de inhame (Dioscorea $s p$ ), araruta (Maranta arundinacea), cará (Dioscorea alata), jalapa (Operculina macrocarpa L. Urban) e feijão verde (Phaseolus vulgaris sp). ABQ RN 2007. [Google Scholar]

[9] Lacerda LG, Colman TAD, Bauab T, Carvalho Filho MAS, Vasconcelos EC, Schnitzler E. Thermal, structural and rheological properties of starch from avocado seeds (Persea americana, Miller) modified with standard sodium hypochlorite solutions. J. Therm. Anal. Calorim. 2014:115(2):1893-1899. [Google Scholar] [Visualizar Item] [CrossRef]

[10]Cereda MP, Vilpoux OF. Tecnologia, usos e potencialidades de tuberosas amiláceas Latino Americanas. Campinas: Fundação Cargill; 2003. [Google Scholar]

[11] Aggarwal P, Dollimore DA. Thermal analysis investigation of partially hidrolyzed starch. Thermochim. Acta. 1998:319(1-2):17-25. [Google Scholar] [Visualizar Item] [CrossRef]

[12] Qianqian J, Wenyuan G, Li X, Zhang J. Characteristics of native and enzymatically hydrolyzed Zea mays L., Fritillaria ussuriensis Maxim. and Dioscorea opposita Thunb. starches. Food Hydroc. 2011:25(3):521-528. [Google Scholar] [Visualizar Item] [CrossRef]

[13] Lacerda LG, Almeida RR, Demiate IM, Carvalho Filho MAS, Vasconcelos EC, Woiciechowski AL, Bannach G, Schnitzler E, Soccol CR. Thermoanalytical and starch content evaluation of cassava bagasse as agro-industrial residue. Braz. Arch. Biol. Tech. 2009:52(spe):143-150. [Google Scholar] [Visualizar Item] [CrossRef]

[14] Oliveira CS, Andrade MMP, Colman TAD, Costa FJOG, Schnitzler E. Thermal, structural and rheological behaviour of native and modified waxy corn starch with hydrochloric acid at different temperatures. J. Therm. Anal. Calorim. 2014:115(1):13-18.

[Google Scholar] [Visualizar Item] [CrossRef]

[15] Weber FH, Collares-Queiroz FP, Chang YK. Caracterização físico-química, reológica, morfológica e térmica dos amidos de milho normal, ceroso e com alto teor de amilose. Cienc. Tecnol. Aliment. 2009:29(4):748-753. [Google Scholar] [Visualizar Item] [CrossRef] 
[16] Chen Y, Huang S, Tang Z, Chen X, Zhang Z. Structural changes of cassava starch granules hydrolyzed by a mixture of $\alpha$-amylase and glucoamylase. Carbohyd. Pol. 2011:85(1):272-275. [Google Scholar] [Visualizar Item] [CrossRef]

[17] Chung H, Lim HS. Effect of partial gelatinization and retrogradation on the enzymatic digestion of waxy rice starch. J. Cereal. Sci. 2006:43:353-359. [Google Scholar] [Visualizar Item] [CrossRef]

[18] Li W, Xiao X, Guo S, Ouyang S, Luo Q, Zheng J, Zhang G. Proximate Composition of Triangular Pea, White Pea, Spotted Colored Pea, and Small White Kidney Bean and Their Starch Properties. Food. Bioprocess. Technol. 2014:7(4):1078-1087. [Google Scholar] [Visualizar Item] [CrossRef]

[19] Naguleswaran S, Vasanthan T, Hoove R, Bressler D. The susceptibility of large and small granules of waxy, normal and high-amylose genotypes of barley and corn starches toward amylolysis at sub-gelatinization temperatures. Food. Res. Int. 2013:51:771-782. [Google Scholar] [Visualizar Item] [CrossRef]

[20] Cai L, Shi Y. Structure and digestibility of crystalline short-chain amylose from debranched waxy wheat, waxy maize, and waxy potato starches. Carbohyd. Pol. 2010:79(4):1117-1123. [Google Scholar] [Visualizar Item] [CrossRef]

[21] Zhang P, Hamaker BR. Banana starch structure and digestibility. Carbohyd. Pol. 2012:87(2):15521558. [Google Scholar] [Visualizar Item] [CrossRef]

[22] Kim H, Huber KC. Physicochemical properties and amylopectin fine structures of A- and B-type granules of waxy and normal soft wheat starch. J. Cereal. Sci. 2010:51(3):256-264.

[Google Scholar] [Visualizar Item] [CrossRef] 